\title{
Scaling, topological tunneling and actions for weak coupling DWF calculations
}

\author{
Greg McGlynn*, Robert D. Mawhinney \\ Physics Department, Columbia University, New York, NY 10027, USA \\ E-mail: gem2128@columbia . edu, rdmephysics . columbia . edu
}

\begin{abstract}
We present results from a $2+1$ flavor DWF calculation at $a^{-1}=3 \mathrm{GeV}$ and discuss strategies for similar calculations at finer lattice spacings which will target charm physics. At weak coupling the autocorrelation time of the global topological charge becomes very long because the HMC algorithm has trouble moving between topological sectors. We report the results of simulations that test two ideas for reducing the autocorrelation time of topological charge. In weak coupling quenched simulations we find that the open boundary conditions suggested by Lüscher and Schaefer do not prevent the appearance of extremely long autocorrelation times for topological observables. We discuss the idea of a "dislocation-enhancing determinant" and show that it can produce an increase in topological tunneling.
\end{abstract}

31st International Symposium on Lattice Field Theory LATTICE 2013

July 29. August 3, 2013

Mainz, Germany

\footnotetext{
*Speaker.
} 


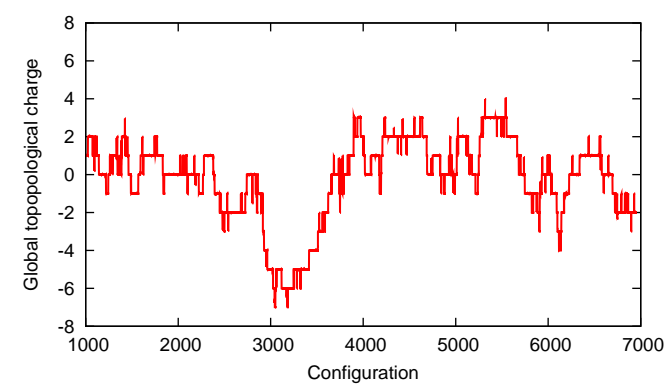

(a) Topological charge

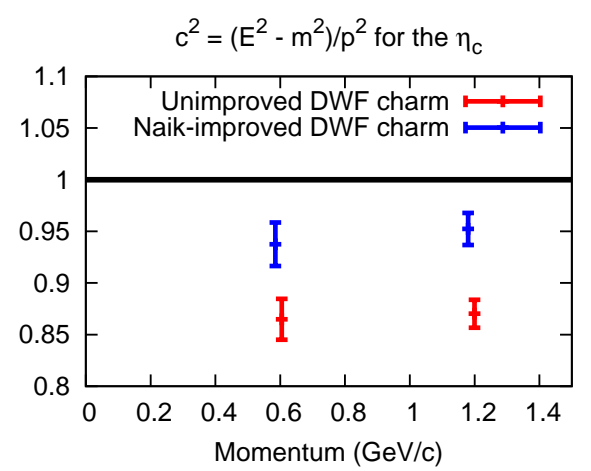

(b) $\eta_{c}$ dispersion relation

Figure 1: Measurements on a 2+1 flavor lattice QCD simulation with $a=0.065(2) \mathrm{fm}$ and $m_{\pi} \approx 360 \mathrm{MeV}$.

\section{Motivation}

Long autocorrelations in Monte Carlo simulations make it hard to reliably estimate statistical errors on measured quanitites. In lattice QCD simulations, the autocorrelation time of the topological charge increases alarming as the lattice spacing is reduced [1]. Depending on the gauge action, at lattice spacings of order $0.05 \mathrm{fm}$ and below the autocorrelation time of the topological charge can be comparable to the typical length of a lattice QCD simulation.

As an example of the problem with topology, Figure 1a shows the topological charge $Q$ as a function of MD time for a QCD simulation with 2+1 flavors of domain-wall quarks at $a=0.065(2)$ $\mathrm{fm}$. The integrated autocorrelation time (IAT) of $Q$ is of order $250 \mathrm{MD}$ time units. While this might be (barely) tolerable, the autocorrelation time is expected to grow very quickly as $a$ decreases, such that the IAT will soon be comparable to or greater than the length of the simulation itself. For example at $a=0.05 \mathrm{fm}$ the autocorrelation time of $Q$ would probably be unacceptably long and we would have to worry about whether statistical errors could be estimated reliably.

But we would very much like to simulate at $a \lesssim 0.05 \mathrm{fm}$ in order to reduce the discretization errors associated with physical-mass charm quarks. Figure $1 \mathrm{~b}$ shows $c^{2} \equiv\left(E^{2}-m^{2}\right) / p^{2}$ for the physical-mass $\eta_{c}$ meson measured on the $a=0.065 \mathrm{fm}$ ensemble with domain-wall valence charm quarks. The $\sim 13 \%$ difference from the correct value $c^{2}=1$ is a measure of $O(4)$ symmetry breaking due to lattice artifacts. This is reduced to $\sim 7 \%$ with Naik improvement, and going to $a=0.05 \mathrm{fm}$ would bring these $O\left(a^{2}\right)$ discretization errors below $5 \%$, which would be very helpful in taking the continuum limit. Another motivation for decreasing $a$ is that finer lattice spacings will also allow for better matching of lattice simulations to perturbation theory by allowing the matching to be performed at a higher energy scale where perturbation theory is more reliable.

Therefore it would be very useful to find some way to reduce the autocorrelation time of the topological charge in weak coupling lattice QCD simulations. We discuss results from experiments to test two ideas for reducing the autocorrelation time of $Q$ : open boundary conditions and a "dislocation-enhancing determinant." 


\begin{tabular}{c|c|c} 
Gauge action & Iwasaki & Wilson \\
\hline \hline$\beta$ & 2.9 & 6.42 \\
\hline$a$ & $0.069(2) \mathrm{fm} \mathrm{[3]}$ & $0.0500(4) \mathrm{fm} \mathrm{[4]}$ \\
\hline Lattice volume & $24^{3} \times 64$ & $32^{3} \times 32$ \\
\hline Physical volume & $(1.7 \mathrm{fm})^{3} \times(4.4 \mathrm{fm})$ & $(1.6 \mathrm{fm})^{4}$ \\
\hline MD time units & 18000 & 5300 \\
\hline
\end{tabular}

Table 1: Parameters of the open boundary conditions experiments. The Wilson ensemble parameters were chosen to coincide with one of the runs in [2].

\section{Open boundary conditions}

Lüscher and Schaefer have proposed using open boundary conditions in the time direction to reduce the autocorrelation time of $Q$ [2]. With the usual periodic boundary conditions, if the gauge field is smooth (as it is at small $a$ ), $Q$ is quantized and so can only change by "tunneling" between disconnected sectors of the field space. These tunneling events are rare at small $a$. But if we choose open boundary conditions in at least one direction, then $Q$ is no longer quantized and can change continuously as topological charges flows in or out through the lattice boundaries, without the need for tunneling events. This may let $Q$ change more quickly, reducing its autocorrelation time.

We ran experiments with two different gauge actions to test whether open boundary conditions indeed reduce the autocorrelation time of $Q$ at weak coupling. We simulated pure SU(3) gauge theory with the HMC algorithm. (We are ultimately interested in improving simulations with dynamical fermions, which use the HMC algorithm, so our pure gauge theory experiments use HMC rather than, say, a heat bath update algorithm). Table 1 describes the parameters of each simulation. In each experiment we compared a reference ensemble with periodic boundary conditions to an otherwise identical ensemble with open boundary conditions in time direction.

When open boundary conditions are used, the simulated physics is distorted in a layer near the open boundaries. For instance, the mean plaquette is different near the boundaries than in the bulk of the lattice (by "bulk" we mean the interior region of the lattice far from the boundaries where the physics is independent of the boundary conditions). We are careful to compare the open and periodic lattices only in the bulk. The boundary region to be excluded has a width that is determined by two main effects. First, the influence of the boundary should be expected to penetrate a distance into the bulk of order $1 / m$ where $m$ is the mass of the lightest state in the theory. In the pure gauge theory this is a glueball with a mass $m \sim 1 \mathrm{GeV}$, so that $1 / m$ is of order 3-4 lattice spacings at our values of $a$. Second, if cooling algorithms such as link smearing or the Wilson flow are used, they effectively average the gauge field over some radius and thus extend the region that should be excluded because of the influence of boundary effects.

Because of these boundary effects, we do not want to compare the global topological charge $Q$ between the open and periodic lattices. The next best thing is to construct a subvolume topological charge by summing the topological charge density over a large interior region of the lattice:

$$
Q\left(t_{1}, t_{2}\right) \equiv \sum_{t_{1} \leq t<t_{2}} \sum_{\vec{x}} \rho(\vec{x}, t)
$$




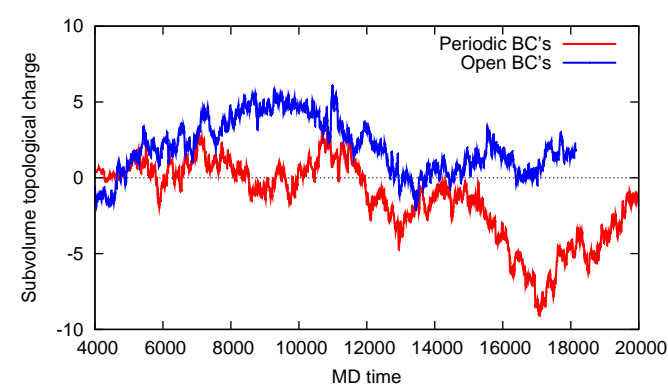

(a) Iwasaki, $\beta=2.9$

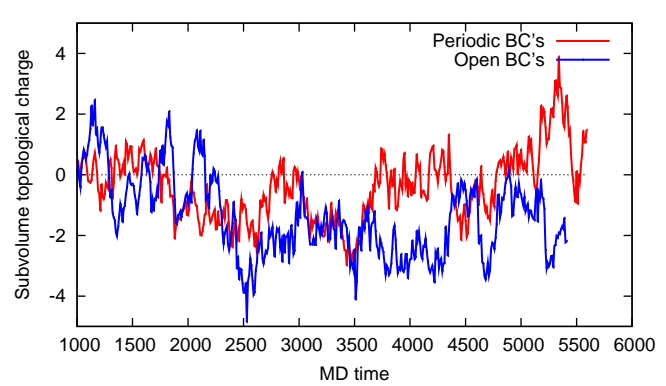

(b) Wilson, $\beta=6.42$

Figure 2: MD time history of the topological charge summed over large subvolumes. On the $24^{3} \times 64$ Iwasaki ensembles we plot $Q(16,48)$ (a sum over the central 32 time slices), while on the $32^{4}$ Wilson ensembles we plot $Q(8,24)$ (a sum over the central 16 time slices).

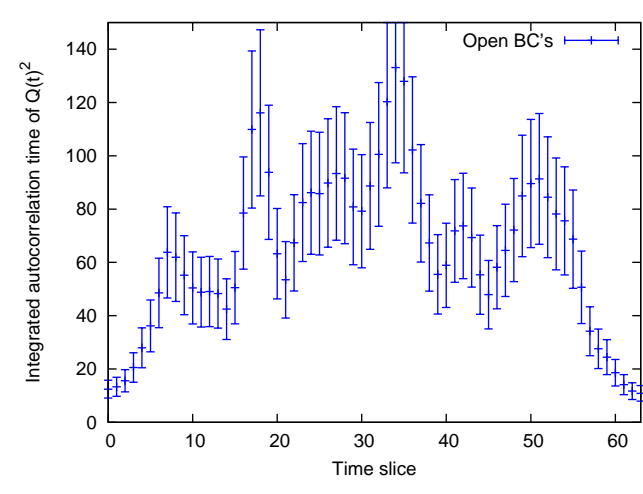

(a) Iwasaki, $\beta=2.9$

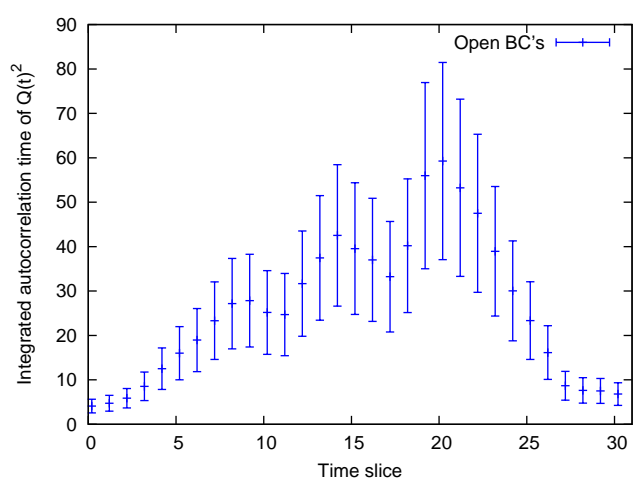

(b) Wilson, $\beta=6.42$

Figure 3: Measured integrated autocorrelation time of $Q(t)^{2}$ as a function of $t$ on open lattices. The integration window was $250 \mathrm{MD}$ time units on the Iwasaki ensemble and $150 \mathrm{MD}$ time units on the Wilson ensemble. Note that IATs in the bulk are likely to be significantly underestimated beyond what is suggested by the error bars because of the relatively short length of our simulations.

where $t_{1}$ and $t_{2}$ must be chosen to exclude the boundary regions. For the topological charge density $\rho(\vec{x}, t)$ we use the 5Li definition from [5], measured after 60 rounds of APE smearing with coefficient 0.45 . As mentioned above, link smearing increases the size of the boundary region. Our parameters produce an RMS smearing radius of order 6 lattice spacings [6].

For large subvolumes we observe very long autocorrelation times of the subvolume charge, independent of the boundary conditions used. An example is shown in Figure 2. While the autocorrelation times are much too long for us to be able to make reliable numerical estimates, they are clearly of order thousands of MD time units on the Iwasaki ensembles and at least many hundreds on the Wilson ensembles. Visually, the time histories for the open lattices do not look any better than those for the periodic lattices.

The open boundaries do have a dramatic effect on autocorrelation times within a narrow region near the boundaries. In order to measure the effect of the open boundaries as a function of the Euclidean time coordinate we can compute $Q(t) \equiv Q(t, t+1)$, the topological charge summed over a single time slice. Figure 3 shows the integrated autocorrelation time of $Q(t)^{2}$ as a function of $t$ 
on the open lattices. It is clear that open boundary conditions reduce the integrated autocorrelation time of $Q(t)^{2}$ dramatically for time slices near the time boundaries compared to time slices in the bulk.

However what we really care about is improving autocorrelation times in the interior region of the lattice, where the physics is independent of the boundary conditions. When we measure $Q(t)^{2}$ on the periodic ensembles, we see no difference between IATs on the periodic lattices and the IATs in the bulk region of the corresponding open lattices. However, we caution that our runs are far too short to estimate these integrated autocorrelation times reliably, so that this comparison cannot be taken too seriously. The autocorrelation function of $Q(t)^{2}$ likely has a long tail which we cannot measure well, so that the integrated autocorrelation time is likely to be significantly underestimated ${ }^{1}$.

Since we lack sufficient statistics to do a numerical comparison of autocorrelation times, it is possible that open boundary conditions are producing some improvement. But Figure 2 shows that even if autocorrelation times in the interior region far from the boundary are shorter with open boundary conditions, they are still unacceptably long.

\section{Dislocation-enhancing determinant}

Since a smooth gauge field cannot change its value of $Q$ continuously, lattice simulations must "tear" the gauge field when they move between topological sectors. These tears or "dislocations" are associated with zero eigenvalues of the hermitian Wilson Dirac operator $H(M)$ for mass $M \sim$ $-1 / a$. For example, each change in the index of the overlap Dirac operator is associated with an eigenvalue of $H(M)$ passing through zero [7]. (We don't measure $Q$ from the index of the overlap Dirac operator, but all definitions of $Q$ should be equivalent in the continuum limit.)

This suggests that we might increase the rate of transitions between topological sectors by encouraging more zero-modes of $H(M)$ for $M \sim-1 / a$. The intuition is that there is an action barrier in the space of lattice gauge fields between different topological sectors and that we can increase the rate of tunneling through the barrier by decreasing the barrier height. In the continuum limit dislocations will become vanishingly rare no matter what we do, but at finite lattice spacing we can increase their density in the hope of reaching smaller $a$ before topology freezes. To enhance dislocations we can introduce an auxiliary determinant into the lattice action:

$$
\operatorname{det} f(H(M))=\prod_{i} f\left(\lambda_{i}\right)
$$

where $\left\{\lambda_{i}\right\}$ are the eigenvalues of $H(M)$. The nonnegative function $f(\lambda)$ should go to 1 for large $\lambda$ so that the actual QCD physics is not affected. But the value of $f(\lambda)$ near $\lambda=0$ can be adjusted to control the density of dislocations.

In the past people have usually instead tried to suppress the zero-modes of $H(M)$, for example because these modes increase the residual chiral symmetry breaking in the domain-wall formulation of chiral fermions. If $0 \leq f(0)<1$ then dislocations are suppressed. For example the

\footnotetext{
${ }^{1}$ We thank Martin Lüscher and Stefan Schaefer for useful discussions on this point.
} 
RBC-UKQCD collaboration has sucessfully used the "dislocation-suppressing determinant ratio"

$$
f(\lambda)=\frac{\lambda^{2}+\varepsilon_{f}^{2}}{\lambda^{2}+\varepsilon_{b}^{2}}
$$

with $\varepsilon_{f} \ll \varepsilon_{b}$ to reduce the residual chiral symmetry breaking in DWF simulations at strong coupling [8]. At weak coupling the residual chiral symmetry breaking is already small and we can instead contemplate making $f(0)>1$ in order to enhance dislocations and speed up topological tunneling. We call this a "dislocation-enhancing determinant" (DED).

Perhaps the most straightforward way of doing this is to use Eq. (3.2) with $\varepsilon_{f}>\varepsilon_{b}$. This ratio is easily simulated using twisted-mass Wilson fermions. But we found that if we introduced a DED using this form of $f$ we needed to increase $\beta$ substantially in order to keep the lattice spacing unchanged. Increasing $\beta$ tends to suppress topological tunneling and so we did not find much net improvement in the tunneling rate from this form of $f$.

However there is a great deal of freedom to choose the form of $f$. We sought an $f(\lambda)$ that went to zero very rapidly for large $\lambda$ in an attempt to avoid affecting as many high modes of $H(M)$, which we expected would reduce the $\beta$ shift needed to maintain the lattice spacing. We chose

$$
f(\lambda)=\left(1-\frac{A}{\lambda^{2}+B_{1}}+\frac{A}{\lambda^{2}+B_{2}}\right)^{2}
$$

With this form $f(\lambda)=1+O\left(\lambda^{-4}\right)$ for large $\lambda$, which is a faster falloff than the form of Eq. (3.2) where $f(\lambda)=1+O\left(\lambda^{-2}\right)$. This determinant is easily simulated using existing RHMC codes. The constants $A, B_{1}$, and $B_{2}$ can be tuned to control the zero-mode enhancement $f(0)$ and the width of the region around zero in which eigenvalues are enhanced. For convenience in our simulations we actually replace $H(M)$ with the hermitian even-odd preconditioned Wilson Dirac operator

$$
H_{\text {prec }}(M)=\gamma_{5}\left((M+4)+\frac{1}{M+4} D_{o e} D_{e o}\right)
$$

where $D_{o e}$ and $D_{e o}$ are the parts of the Wilson Dirac operator that connect sites of opposite parity. If $H$ has a zero eigenvalue then so does $H_{\text {prec }}$, so this still enhances dislocations.

Figure 4 shows the topological charge measured in a quenched simulation using this DED with the Wilson gauge action at $a=0.05 \mathrm{fm}$. Also shown for reference is the topological charge evolution on an ensemble at the same lattice spacing using the regular unmodified Wilson gauge action. It is important to make such comparisons at the same lattice spacing, since the topological tunneling rate depends very sensitively on $a$. The lattice spacings for the two ensembles were matched using the Wilson flow scale $t_{0}$ [9] by adjusting the value of $\beta$ on the DED ensemble. This required increasing $\beta$ to 6.70 on the DED ensemble compared to 6.42 on the reference ensemble.

Although the problem of long autocorrelations has by no means been solved, the DED clearly improves the topological tunneling rate in Figure 4. The frequency with with $Q$ changes is about 5 times greater in the DED ensemble compared to the reference ensemble with the unmodified Wilson gauge action. Furthermore, the DED ensemble explores a much wider range of topological sectors in the same span of MD time. It is possible that even greater gains could be achieved with a different form of $f$, or by using a different value of $M$. 


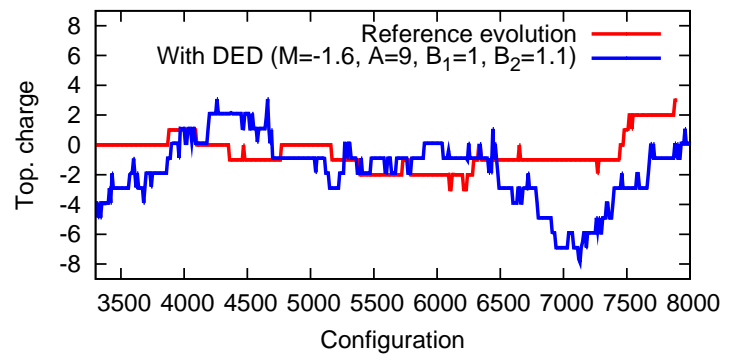

Figure 4: Topological charge as a function of MD time measured on a DED ensemble and a reference ensemble at $a=0.05 \mathrm{fm}$.

\section{Conclusions}

We have investigated two techniques intended to reduce the autocorrelation time of the topological charge in lattice QCD simulations at weak coupling. In our experiments with open boundary conditions, we find unacceptably long autocorrelations for the charge in large subvolumes independent of the boundary conditions. The long autocorrelations combined with the limited length of our runs unfortunately prevents us from making a direct numerical comparison of autocorrelation times between open and periodic boundary conditions. We do find some success in increasing the rate of topological tunneling by introducing a dislocation-enhancing determinant, although so far the autocorrelations are still longer than we would like.

\section{References}

[1] S. Schaefer et al. [ALPHA Collaboration], "Critical slowing down and error analysis in lattice QCD simulations," Nucl. Phys. B 845, 93 (2011) [arXiv:1009.5228 [hep-lat]].

[2] M. Lüscher and S. Schaefer, "Lattice QCD without topology barriers," JHEP 1107, 036 (2011) [arXiv:1105.4749 [hep-lat]].

[3] A. Ali Khan et al. [CP-PACS Collaboration], "Kaon B parameter from quenched domain wall QCD," Phys. Rev. D 64, 114506 (2001) [hep-lat/0105020].

[4] S. Necco and R. Sommer, "The $N_{f}=0$ heavy quark potential from short to intermediate distances," Nucl. Phys. B 622, 328 (2002) [hep-lat/0108008].

[5] P. de Forcrand, M. Garcia Perez and I.-O. Stamatescu, "Topology of the SU(2) vacuum: A lattice study using improved cooling,” Nucl. Phys. B 499, 409 (1997) [hep-lat/9701012].

[6] C. W. Bernard and T. A. DeGrand, "Perturbation theory for fat link fermion actions," Nucl. Phys. Proc. Suppl. 83, 845 (2000) [hep-lat/9909083].

[7] R. Narayanan and H. Neuberger, "Chiral determinant as an overlap of two vacua," Nucl. Phys. B 412, 574 (1994) [hep-lat/9307006].

[8] R. Arthur et al. [RBC and UKQCD Collaborations], "Domain wall QCD with near-physical pions," Phys. Rev. D 87, 094514 (2013) [arXiv:1208.4412 [hep-lat]].

[9] M. Lüscher, “Properties and uses of the Wilson flow in lattice QCD," JHEP 1008, 071 (2010) [arXiv:1006.4518 [hep-lat]]. 